\title{
Satisfacción de los pacientes atendidos en una clínica odontológica de la Universidad Cooperativa de Colombia
}

\author{
Ángela María Sarrazola-Moncada*, MSc ${ }_{1}$, Melissa Andrea Castaño-Tabares, \\ Est., $\mathrm{OD}_{1}$, Andrea Sánchez-Upegui, Est., $\mathrm{OD}_{1}$, María Alejandra García- \\ Moreno, Est., $\mathrm{OD}_{1}$, Milena Arévalo, Est., $\mathrm{OD}_{1}$, Yenifer Peláez, Est., $\mathrm{OD}_{1}$
}

Universidad Cooperativa de Colombia, sede Medellín y Envigado, Antioquia, Colombia

Recibido: 25 de agosto del 2015 Aprobado: 21 de octubre del 2015

*Autor de correspondencia: Ángela María Sarrazola Moncada. Docente Facultad de Odontología Universidad Cooperativa de Colombia, sede Medellín y Envigado, Carrera 42 n. ${ }^{\circ}$ 49-95, Bloque 8, Medellín, Antioquia, Colombia. Teléfono: (57) 4 4446065. Correo electrónico: angela.sarrazola@campusucc.edu.co

Cómo citar este artículo: Sarrazola-Moncada ÁM, Castaño-Tabares ME, Sánchez-Upegui A, García-Moreno MA, Arévalo M, Peláez Y. Satisfacción de los pacientes atendidos en una clínica odontológica de la Universidad Cooperativa de Colombia. Rev Nac Odontol. 2016;12(23):57-62. doi: 10.16925/od.v12i23.1378

Resumen. Introducción: la satisfacción de los pacientes con los servicios de salud recibidos es un elemento fundamental en la medición de la calidad de la atención. Objetivo: describir la satisfacción de los pacientes que consultaron en la clínica del adulto de la Facultad de Odontología de la Universidad Cooperativa de Colombia, sede Medellín y Envigado, Colombia. Materiales y métodos: estudio descriptivo transversal. Se calculó una muestra representativa de 188 pacientes que se encuestaron aleatoriamente, previo consentimiento informado. Se hizo un análisis descriptivo. Resultados: $68,1 \%$ fueron mujeres, la edad promedio fue de 34,04 \pm 9,71 años. El 68\% consultó para prótesis fija, $16,5 \%$ para prótesis removible, $14,4 \%$ para prótesis total y $1,1 \%$ para blanqueamiento dental. El $100 \%$ de la población atendida informa que recibió buen trato del estudiante, el profesor y el auxiliar de clínica. Todos los pacientes consideraron que fue solucionado su motivo de consulta. El 95,7\% de los pacientes volvería a consultar y el 99\% recomendaría el servicio de odontología. La opinión de los pacientes respecto a las instalaciones físicas fue buena en general para la higiene e iluminación (97\%), el orden (96\%) y la ventilación (83\%). La privacidad fue el único factor evaluado como malo (3,2\%). Conclusiones: los pacientes atendidos en la clínica integral del adulto quedaron satisfechos con la atención recibida y su motivo de consulta fue resuelto; consultaron principalmente por tratamiento de prótesis fija y parcial removible; la mayoría regresaría, recomendaría el servicio a otras personas y tiene una opinión excelente respecto a las relaciones interpersonales y buena en cuanto a la planta física, excepto por la privacidad.

Palabras clave: calidad de servicios de salud, estudiantes de odontología, Facultad de Odontología, prácticas clínicas, satisfacción. 


\title{
Satisfaction of the Patients cared for in the Dental Clinic of the Cooperative University of Colombia
}

\begin{abstract}
Introduction: The patients' satisfaction of the health services received is a key element in the measurement of the quality of the attention. Objective: To describe the patient's satisfaction that received care at the adult clinic of the Dental School of the Cooperative University of Colombia, in the facility of Medellin and Envigado, Colombia. Materials and Methods: Cross-sectional descriptive study. A representative sample was estimated from 188 patients that were randomly surveyed, given previous informed consent. A descriptive analysis was performed. Results: $68.1 \%$ were women with an average age of $34.04 \pm 9.71$ years. $68 \%$ requested consultation for fixed prosthesis, 16 , and $5 \%$ for removable dentures, $14.4 \%$ for total prostheses and $1.1 \%$ for teeth whitening. $100 \%$ of the population served informs they received good treatment from the student, professor and the clinic staff. All the patients consider their reason for consultation was solved. 95, 7\% of the patients would consult at that clinic again and 99\% would recommend the dental services. The patient's perception regarding the physical installations was generally high for hygiene and illumination (97\%), the order (96\%) and ventilation (83\%). Privacy was the only factor assessed poorly (3.2\%). Conclusions: The patients treated at the integral adult clinic were satisfied with the attention received and their reason for consultation was resolved. The patients mainly requested consultation for the treatment of fixed or partially removable prosthesis. Most of them agreed to return and recommend the clinic's service to third parties, and have an excellent opinion regarding interpersonal relationships, and a good opinion regarding the physical installations, except for the patient's privacy.
\end{abstract}

Keywords: quality of health services, dental students, dental school, clinical practices, satisfaction.

\section{Satisfação dos pacientes atendidos em clínica odontológica da Universidade Cooperativa de Colômbia}

Resumo. Introdução: a satisfação dos pacientes com os serviços de saúde recebidos é um elemento fundamental na medição da qualidade de atendimento. Objetivo: descrever a satisfação dos pacientes que consultaram na clínica do adulto da Faculdade de Odontologia da Universidade Cooperativa de Colômbia, sede de Medellín e Envigado, Colômbia. Materiais e métodos: estudo descritivo transversal. Calculou-se uma amostra representativa de 188 pacientes que foram pesquisados de forma randomizada, prévio consentimento informado. Realizou-se uma análise descritiva. Resultados: 68,1\% foram mulheres, a idade média foi de 4,04 $\pm 9,71$ anos. $68 \%$ consultou para prótese fixa, $16,5 \%$ para próteses removível, $14,4 \%$ para próteses total e $1,1 \%$ para clareamento dental. $100 \%$ da população atendida indica que recebeu bom tratamento do estudante, o professor e o ajudante de clínica. Todos os pacientes consideraram que foi resolvido seu motivo de consulta. 95,7\% dos pacientes consultaria novamente e $99 \%$ recomendaria o serviço de odontologia. A opinião dos pacientes em relação às instalações físicas foi boa em geral para a higiene e iluminação (97\%), a organização (96\%) e a ventilação ( $83 \%)$. A privacidade foi o único fator avaliado como ruim $(3,2 \%)$. Conclusões: os pacientes atendidos na clínica integral do adulto estiveram satisfeitos com o atendimento recebido e seu motivo de consulta foi resolvido; consultaram principalmente por tratamento de prótese fixa e parcial removível; a maioria voltaria, recomendaria o serviço para outras pessoas e tem uma opinião excelente em relação às relações interpessoais e boa em relação à infraestrutura física, menos pela privacidade.

Palavras chave: qualidade de serviços de saúde, estudantes de odontologia, Faculdade de Odontologia, práticas clínicas, satisfação. 


\section{Introducción}

La Organización Mundial de la Salud (oms) considera que la calidad de los servicios de salud debe contemplar los siguientes atributos: excelencia profesional, uso eficiente de los recursos, mínimo de riesgos para el paciente y alto grado de satisfacción del paciente [1].

En el marco del Sistema Obligatorio de la Garantía de la Calidad (sogc Decreto 1011 del 2006), que es parte del Sistema General de Seguridad Social en Salud, se requiere hacer una evaluación continua para el mejoramiento de la calidad observada respecto a la calidad esperada en la atención en salud, con el propósito de lograr la adhesión y satisfacción de los pacientes [2]. Es así como la satisfacción de los pacientes es fundamental en la evaluación de la calidad de los servicios. Una de las formas de conocerla es mediante su indagación, la cual se construye durante el proceso de prestación del servicio de salud $[3,4]$.

El paciente que acude a la Facultad de Odontología de la Universidad Cooperativa de Colombia llega con una necesidad de atención, expectativas y deseos, característica propia de cualquier paciente que solicita un servicio de salud bucal y que espera que su motivo de consulta sea solucionado para recuperar su salud bucal [5]. Sin embargo, el marco de su atención va a ser diferente y desencadena otros retos adicionales; es recibido en una clínica de la facultad, donde está inmerso en un modelo de atención docencia-servicio, en el cual intervienen el estudiante y el profesor en la prestación del servicio. Esto hace que la relación que se establezca y los procesos que se efectúen tengan unas características diferentes a las de cualquier otro servicio en salud; la atención por personal en formación hace que este modelo de atención influya de modo importante en la calidad, dado que es diferente que el procedimiento odontológico lo haga un profesional a que intervengan el estudiante y el profesor.

La Universidad, hoy más que nunca con su enfoque de acreditación y proyección social, tiene la responsabilidad de establecer el nivel de satisfacción de los pacientes, con el propósito de retroalimentar sus procesos de atención, no solo desde el punto de vista técnico-científico, sino también a partir de las relaciones interpersonales [6].

En el 2006, en un estudio desarrollado en una institución universitaria prestadora de servicios de salud se encontró: baja satisfacción en cuanto a la percepción de los pacientes hacia la calidad de los servicios, insatisfacción con respecto a las necesidades de los estudiantes y alta insatisfacción en lo referente a sus expectativas [7].

El presente estudio describe la satisfacción de los pacientes con la atención odontológica en las clínicas del adulto de la Facultad de Odontología de la Universidad Cooperativa de Colombia, sede Medellín y Envigado.

\section{Materiales y métodos}

Se hizo un estudio observacional, descriptivo y transversal. A partir de un histórico de atención del semestre 2014-1 de 370 pacientes, se calculó una muestra representativa de 188 pacientes, con una confianza del $95 \%$, un error de muestreo del $5 \%$ y una proporción del $50 \%$.

Aleatoriamente, previa prueba piloto y diligenciamiento del consentimiento informado (estudio sin riesgo según Resolución 8430 de 1993), se encuestó a los pacientes mayores de edad que consultaron durante el semestre 2014-2 en la Facultad de Odontología de la Universidad Cooperativa de Colombia, sede Medellín y Envigado.

A fin de procesar la información, se creó una base de datos en Microsoft Excel y se verificó la validez de la información digitada corroborando aleatoriamente la información de las encuestas suministrada por los pacientes; luego, se hizo un análisis univariado, se calcularon frecuencias y porcentajes para las variables cualitativas y promedios con desviación estándar para las variables cuantitativas. Se utilizó el programa estadístico SPSS versión 21.0.

\section{Resultados}

El $68,1 \%$ (128) de las personas evaluadas fueron mujeres. El promedio de edad de los encuestados fue de 34,04 \pm 9,71 (20-76) años. El 39,9\% ha realizado estudios de secundaria, el $41,5 \%$ reside en estrato tres y el $50 \%$ procede de la ciudad de Medellín. Los pacientes conocían de la prestación del servicio en la Facultad de Odontología principalmente por amigos $(34,6 \%)$ y familiares $(26,6 \%)$ (tabla 1$)$. 
Tabla 1. Características sociodemográficas de los pacientes participantes en el estudio

\begin{tabular}{|c|c|c|}
\hline Variable & $\%$ & (n) \\
\hline \multicolumn{3}{|l|}{ Sexo } \\
\hline Mujeres & 68,1 & 128 \\
\hline Hombres & 31,9 & 60 \\
\hline \multicolumn{3}{|l|}{ Procedencia } \\
\hline Medellín & 50 & 94 \\
\hline Envigado & 20,7 & 39 \\
\hline Itagüí & 14,8 & 28 \\
\hline Otros $^{*}$ & 14,4 & 27 \\
\hline \multicolumn{3}{|l|}{ Nivel de escolaridad } \\
\hline Secundaria & 63,3 & 119 \\
\hline Primaria & 20,2 & 38 \\
\hline Tecnología & 9,0 & 17 \\
\hline Universitario & 7,4 & 14 \\
\hline \multicolumn{3}{|l|}{ Seguridad social } \\
\hline Régimen contributivo & 64,4 & 121 \\
\hline Régimen subsidiado & 35,1 & 66 \\
\hline No asegurado & 0,5 & 1 \\
\hline \multicolumn{3}{|l|}{ Estrato socioeconómico } \\
\hline Bajo (1-2) & 36,7 & 69 \\
\hline Medio (3-4) & 54,3 & 102 \\
\hline Alto (5-6) & 9 & 17 \\
\hline
\end{tabular}

${ }^{*}$ Otros: Barbosa, Guarne, Titiribí, Frontino, Rionegro,

Copacabana y Sabaneta

Fuente: elaboración propia

El $68 \%$ asistió a la consulta por tratamiento de prótesis fija, el $16,5 \%$ por prótesis removible, el $14,4 \%$ por prótesis total y $1,1 \%$ por blanqueamiento dental. El $100 \%$ (188) de los pacientes informó que su motivo de consulta principal fue (o estaba siendo) solucionado. La información suministrada a los pacientes por los estudiantes acerca de su condición de salud y de la necesidad de tratamiento fue clara. El $100 \%$ de la población atendida informa que recibió un buen trato por el estudiante, el docente y el auxiliar de clínica.

En cuanto a la evaluación de la satisfacción general, el $57 \%$ estuvo muy satisfecho, el $39 \%$ estuvo satisfecho y el $4 \%$ estuvo medianamente satisfecho; ninguno reportó insatisfacción. El 95,7\% de los pacientes volvería a consultar, lo que hace referencia a la satisfacción inmediata, y el 99,5\% recomendaría el servicio de odontología, lo cual hace alusión a la satisfacción general. La opinión de los pacientes respecto a las instalaciones físicas fue buena en general para la higiene, la iluminación, el orden y la ventilación. Solo la privacidad fue evaluada como mala por algunos (tabla 2).

Tabla 2. Opinión de los pacientes participantes en el estudio sobre los aspectos físicos

\begin{tabular}{|l|c|c|c|}
\hline Aspecto físico & Buena & Regular & Mala \\
\hline Higiene & $96,8 \%(182)$ & $3,2 \%(6)$ & $0 \%$ \\
\hline Orden & $95,7 \%(180)$ & $4,3 \%(8)$ & $0 \%$ \\
\hline Iluminación & $96,8 \%(182)$ & $3,2 \%(6)$ & $0 \%$ \\
\hline Ventilación & $83,3 \%(166)$ & $11,7 \%(22)$ & $0 \%$ \\
\hline Privacidad & $85,1 \%(160)$ & $11,7 \%(22)$ & $3,2 \%(6)$ \\
\hline
\end{tabular}

Fuente: elaboración propia

\section{Discusión}

Ofrecer un servicio que genere satisfacción es el baluarte esperado de todo prestador de un servicio en salud. En el presente estudio, se encontró satisfacción con la atención recibida en la mayoría de los pacientes ( $96 \%$ ), similar a lo encontrado en una institución educativa en Cartagena, donde el $89 \%$ de los encuestados manifestó estar satisfecho con los servicios recibidos [8].

El 99,5\% de los pacientes que consultan en la Facultad de Odontología de la Universidad Cooperativa, sede Medellín y Envigado, recomendaría el servicio de la facultad. Este resultado es afín al reporte del estudio de Hincapié y colaboradores [9] de la Facultad de Odontología de la Universidad de Antioquia, en el cual el 98,1\% de los pacientes también recomendaría la atención clínica en esa facultad, lo cual corrobora la satisfacción general frente al servicio e indica de cierta manera la efectividad de los esfuerzos de la comunidad académica para prestar un servicio de calidad.

Las interacciones interpersonales tienen implicaciones directas en la gestión de la atención al paciente antes, durante y después del procedimiento. En el presente estudio, se encontró que la comunicación fue clara para todos los participantes, resultado equivalente al encontrado en el estudio de Hincapié y colaboradores [7]. Dicho aspecto es fundamental para generar confianza [10].

En el contexto de la formación del recurso humano, la relación establecida entre docentes y estudiantes también puede influir en la prestación 
del servicio. Tal relación fue percibida de forma favorable, similar a lo encontrado en el estudio realizado en la Facultad de Odontología de la Universidad de Antioquia, en la clínica integral del adulto en el 2013, por López y colaboradores [11, 12].

El factor de privacidad fue uno de los aspectos a mejorar en los resultados, dado que es un elemento crítico en los modelos de atención docencia-servicio, similar a lo encontrado en el estudio de López y colaboradores [8]. En el presente estudio, la privacidad fue evaluada desde el sentido físico, pero se debe tener en cuenta que este factor opera en un ámbito subjetivo y es también mediado por la interacción entre los docentes, los estudiantes y los pacientes en torno a un proceso de enseñanza-aprendizaje, lo cual se puede seguir estudiando desde enfoques cualitativos que faciliten su abordaje en el contexto de la prestación de un servicio de calidad [13].

Finalmente, cabe resaltar que la mayoría de los pacientes, sobre todo mujeres de estrato socioeconómico medio y bajo [14], consultó para tratamientos no contemplados en el plan obligatorio de salud (POS), como prótesis fijas y parciales removibles. Esto evidencia entre los adultos consultas por pérdidas dentarias y acogida de modelos de atención docencia-servicio como una opción de consulta en salud para suplir necesidades de atención odontológica, lo cual aporta a la transformación de la condición de salud bucal de la población, tal como se ha planteado en la política pública de salud bucal de Medellín [15].

\section{Conclusiones}

Los pacientes atendidos en la clínica integral del adulto quedaron satisfechos con la atención recibida y su motivo de consulta fue resuelto; consultaron principalmente por tratamiento de prótesis; la mayoría regresaría, recomendaría el servicio a otras personas, tiene una opinión excelente respecto a las relaciones interpersonales y buena en cuanto a la planta física; excepto por la privacidad para unos pocos pacientes, lo cual no es menos importante.

Es menester del personal directivo y de todos los participantes en el modelo de atención docencia-servicio de la Facultad de Odontología de la Universidad Cooperativa de Colombia, sede Medellín y Envigado, propender a prestar una atención con calidad que satisfaga a los pacientes, tal como fue evaluado, y que mejore la privacidad durante la prestación del servicio de salud bucal en sus clínicas, factor que incluso podría ser abordado no solo desde el componente físico.

\section{Referencias}

[1] World Health Organization. Regional Office for Europe. The principles of quality assurance, report on a wHO meeting. Copenhague: OMS; 1985. En: Varo J. La calidad de la atención médica. Med Clin. 1995;104(14):538-40.

[2] Colombia, Ministerio de la Protección Social. Decreto 1011 de 2006, Por el cual se establece el Sistema Obligatorio de Garantía de Calidad de la Atención de Salud del Sistema General de Seguridad Social en Salud (3 abr 2006).

[3] Ramírez T, Nájera P, Nigenda G. Percepción de la calidad de la atención de los servicios de salud en México: perspectivas de los usuarios. Salud Pública Méx. 1998;40(1):3-12.

[4] Chang WJ, Chang YH. Patient satisfaction analysis: identifying key drivers and enhancing service quality of dental care. J Dent Sci. 2013;8:239-47.

[5] Riley JL, Gordan VV, Hudak-Boss SE, Fellows JL, Rindal B, Gilbert GH. Concordance between patient satisfaction and the dentist's view. JADA. 2014;145(4):355-62.

[6] Universidad Cooperativa de Colombia. Plan Estratégico Nacional 2013-2022 [internet]. 2013. Disponible en: http://www.ucc.edu.co/institucion/ Paginas/plan-estrategico.aspx

[7] Chávez E, Iguarán R, Pérez J. Satisfacción de los clientes del Servicio Médico Odontológico de la Universidad del Zulia. Núcleo Costa Oriental del Lago. Revista Venezolana de Ciencias Sociales. 2006;10(1):176-203 [internet]. Disponible en: http://www.redalyc.org/pdf/309/30910111.pdf

[8] Betin Portacio A, Guardela Simancas A, Infante Franco K, Díaz Caballero A, González Martínez F. Satisfacción de los pacientes respecto a calidad de la atención odontológica en una Institución Pública Universitaria de la ciudad de Cartagena. Revista de la Facultad de Ciencias de la Salud. 2009;6(2):95101.

[9] Hincapié A, Carvajal A, Sánchez LA, Arias Ó. Satisfacción de los usuarios frente a la calidad de la atención en el programa de Odontología Integral del Adolescente y Ortodoncia de la Facultad de Odontología de la Universidad de Antioquia. Rev Fac Odontol Univ Antioq. 2004;15(2):5-11. 
[10] Riley JL, Gordan VV, Rindal B, Fellows JL, Qvist V, Patel S, et al. Components of patient satisfaction with a dental restorative visit. JADA. 2012;143(9):1002-10.

[11] López-Portilla J, Pilataxi-Sánchez S, RodríguezEscobar L, Velásquez-Rivera A, López-Blandón M, Martínez-Delgado C, et al. Determinantes de la satisfacción de la atención odontológica en un grupo de pacientes atendidos en la Clínica del Adulto de la Facultad de Odontología de la Universidad de Antioquia. Rev Gerenc Polit Salud. 2013;12(24):209-25.

[12] Lee KT , Chen CM, Huang ST , Wu YM, Lee HE, Hsu KJ, et al. Patient satisfaction with the quality of dental treatment provided by interns. J Dent Sci. 2013;8:177-83.
[13] Díaz R. Satisfacción del paciente: principal motor y centro de los servicios sanitarios. Revista de Calidad Asistencial. 2002;17(1): 9-22.

[14] Wonik L, Seok-Joo K, Jeffrey MA, Suchitra N. Community factors predicting dental care utilization among older adults. JADA. 2014;145(2):150-8.

[15] Secretaría de Salud de Medellín, Facultad de Salud Pública de la Universidad de Antioquia. La boca convoca. Formulación de la política pública de salud bucal para Medellín 2013-2022 [internet]; 2013. Disponible en: http://politicapublicasaludbucalmed. blogspot.com/2013/02/blog-post_26.html 